\title{
Employing high-end X-ray technologies for laboratory structural biology in Centre of Molecular Structure
}

\author{
J. Stránský ${ }^{1}$ J. Pavlíček ${ }^{1}$, A. Keilbach ${ }^{2}$, J. Dohnálek ${ }^{1}$ \\ ${ }^{1}$ Centre of Molecular Structure, Institute of Biotechnology, CAS v. v. i., Průmyslová 595, Vestec near Prague, ${ }^{2}$ Anton Paar GmbH, \\ Anton-Paar-Strasse 20, Graz \\ Jan.stransky@ibt.cas.cz
}

The Centre of Molecular Structure (CMS) provides services and access to state-of-art instruments, which cover a wide range of techniques required by not only structural biologists. CMS operates as part of the Czech Infrastructure for Integrative Structural Biology (CIISB), and European infrastructures Instruct-ERIC and MOSBRI. CMS is organized in 5 core facilities: CF Protein Production , CF Biophysics, CF Crystallization of proteins and nucleic acids, CF Diffraction techniques, and CF Structural Mass Spectrometry.

CF Diffraction techniques employs two laboratory X-ray instruments equipped with high flux MetalJet X-ray sources: a single crystal diffractometer D8 Venture (Bruker) and a small angle X-ray scattering instrument SAXSpoint 2.0 (Anton Paar). The configurations of both instruments represent top tier of possibilities of laboratory instrumentation. Apart from standard applications, the instruments are also extended for advanced experiments: the diffractometer is equipped with the stage for in-situ crystall diffraction and crystal dehydration, SAXS is equipped with in-situ UV-Vis spectroscopy and liquid chromatography system for SEC-SAXS. The setups enable easy access and fast turn-around of samples under different conditions, but also collection of high quality end-state data without further need for synchrotron data collection in many cases. CF Diffraction provides services in synergy with the other CFs on-site, therefore scientific questions can be quickly answered as they emerge from the experiments.

\section{Keywords: protein; structural biology; X-ray diffraction; small angle X-ray scattering}

The Centre of Molecular Structure is supported by: MEYS CR (LM2018127); project Czech Infrastructure for Integrative Structural Biology for Human Health (CZ.02.1.01/0.0/0.0/16_013/0001776) from the ERDF; UP CIISB (CZ.02.1.01/0.0/0.0/18_046/0015974), and ELIBIO (CZ.02.1.01/0.0/0.0/15_003/0000447). 